\title{
Surface Magnetic Fields in Early-Type Stars
}

\author{
V. S. Airapetian \\ Science Programs, CSC/GSFC, Greenbelt, MD
}

\begin{abstract}
Recent observations imply magnetic activity in atmospheres of early-type stars. We explore the possibility that stressed surface magnetic fields can be driven by inertial oscillations, such as r-modes which are vorticity waves. We show that vorticIAL MOTIOns are able to supply helicity to drive magnetic activity in stellar atmospheres.
\end{abstract}

\section{Introduction}

Stars of spectral types earlier than F5 lack outer convection zones and, therefore, solar-like magnetic activity in terms of atmospheric heating or flare-like transient event should not be expected. However, recent UV and X-ray observations of early-type stars show a variety of features implying magnetic activity in their atmospheres. ROSAT observations of B stars imply that the X-rays from stars later than B2 are magnetically driven (Cohen et. al. 1997a). ASCA X-ray spectroscopy of the B0 V star $\tau$ Sco also suggests magnetic nature of high-energy processes occurring in the stellar atmosphere (Cohen et al. 1997b).

Strong evidence of magnetically controlled processes is also provided by the recent HST/GHRS and ASCA observations of $\gamma$ Cas (Smith et al. 1998, 1999). Similar dark co-rotating features have been observed in Rigel and $\alpha \mathrm{Cyg}$ ) in $\mathrm{H} \alpha$ absorption line (Israelian et al. 1997, Morrison \& Mulliss 1997, Kauffer et al. 1996).

\section{Surface Magnetic Fields Driven by r-modes}

Differential rotation at the interface between the convective core and the radiative zone can drive toroidal magnetic fields creating poloidal currents, so that magnetic field outside will be a potential (current free) field. Moss (1994) has estimated that in order for a flux tube to rise from the convective core to the surface on timescale of $5 \mathrm{Myr}$ or less, magnetic fields up to $10 \mathrm{MG}$ are required. Poloidal surface magnetic fields can be generated or at least redistributed, possibly due to some type of $\alpha$ dynamo implying shearing or twisting of potential magnetic fields. The heating of stellar atmospheres of early-type stars by stressed magnetic fields driven by differential rotation has been considered by Tout \& Pringle (1995). While strong differential rotation can be efficient at the interface between the core and the radiative zone, it is unlikely to occur at the stellar surface because of the lack of interaction of rotation with large-scale or small-scale turbulence (Gilman \& Miller 1984). 
Here we explore the possibility that magnetic fields in atmospheres of hot stars can be stressed by vorticial surface motions in terms of inertial oscillations, such as r-modes. Possible mechanisms of excitations are briefly discussed in the next section. Osaki (1986) has suggested that line-profiles variations of hot stars, such as $\beta$ Cep, can be reproduced by r-modes.

$r$-modes represent incompressible, non-axisymmetric inertial oscillations which exist in convectively stable regions of a rotating star (Saio 1982; Wolff \& Blizard 1986). Because the subadiabatic stratification tends to oppose radial motions, $r$-modes tend to be more confined to two-dimensional horizontal surfaces, and are similar in nature to Rossby waves in the earth's atmosphere. The r-mode oscillations are essentially vorticity waves with characteristic periods exceeding the stellar rotation period. In the two-dimensional (shell) approximation for a slowly rotating star, a linear analysis for an r-mode of spherical harmonic order $m$ and degree $l$ gives a longitudinal propagation rate with respect to the fluid of

$$
\nu_{r}=-\frac{\Omega_{o}}{\pi l(l+1)},
$$

where $\Omega_{0}$ is the mean sidereal angular velocity of the star (Provost et al. 1981; Saio 1982; Wolff 1995). Their non-zero vorticity, particularly at low spherical harmonic degrees, enables them to generate magnetic helicity introducing electric currents in atmospheres of hot stars. The vortex flow drifts westward at the speed $-\nu_{r}$ opposite to the direction of the solar rotation (retrograde). After a half-period the sense of the flow is reversed.The lowest mode $(l=2, m=1)$ with non-zero vorticity can be described as two pairs of vortices. Vortices of a pair are located symmetrically with respect to the solar equator with centers at latitudes of $45^{\circ}$ and $-45^{\circ}$ and rotate in opposite directions (Saio 1982). Transequatorial vorticlAL MOTIOns can twist a potential magnetic field due to twisting of magnetic field lines introducing magnetic helicity. Calculations by Tikhomolov \& Mordvinov (1996) have shown that r-modes in the Sun can induce large-scale variations in the global magnetic field. 3D MHD simulations by Van Hoven et al. (1995) have shown that when horizontal vorticial motions are applied to the bipolar magnetic field, they twist magnetic field lines producing magnetic loops. As twisting (or shearing) angle exceeds a critical one, magnetic loops expand forming a current sheet. The system eventually disrupts through the reconnection process ejecting a plasmoid into the atmosphere (Mikic \& Linker 1994).

If a vorticial motion is applied to the footpoint of a semi-circular loop of length, $L$, it twists longitudinal field lines, $B_{z}$, producing an azimuthal magnetic field $B_{\phi}=B_{z} \frac{C R t}{L}$, where $C$ is the vorticity, $R$ is the loop radius in time $t$. Then, the input surface power associated with vorticial motions in a loop is

$$
P(t)=\frac{t}{8 \pi L}\left(B_{z} C R\right)^{2}
$$

Reconnection occurs at the twist angle of $2 \pi$ (Strauss \& Otani 1988) in a time $t=C^{-1}$. The luminosity of X-ray emission associated with plasma heating driven by reconnection process is

$$
L_{x}=\epsilon \frac{B_{z}^{2} R^{3}}{4 \pi} C .
$$


For a typical Be star, $C=5 \times 10^{-5} \mathrm{~s}^{-1}$ and $R=0.5 \mathrm{R}_{\mathrm{star}}$ we obtain

$$
\left.L_{x}=10^{29} \epsilon B_{z}^{2} \quad \text { (in } \mathrm{ergs} / \mathrm{s}\right)
$$

and we find that for $B_{z} \sim 100 \mathrm{G}$ and $\epsilon \sim 0.01-0.1, L_{x} \sim 10^{31}-10^{32} \mathrm{ergs} / \mathrm{s}$ in agreement with $\mathrm{X}$-ray observations of $\mathrm{Be}$ stars. This process can be efficient only if the life-time of r-modes ( 3 times of the rotation period for the mode considered above) exceeds instability timescale.

\section{Discussion}

We propose that $r$-modes can supply enough mechanical energy to the potential magnetic field forming loops and at certain condition depending on twisting angle can release part of this energy through magnetic reconnection. Future numerical calculation are going to provide details of this process. r-modes can be excited in number of ways as proposed by authors considering their formation in solar interior. Global gravitational oscillations known as g-modes are expected to excite r-modes and likely to exist in early-type stars. Dziembowski (1994) has recently shown that opacity effects in their radiative envelopes can excite gmodes, and he reports possible observational evidence for such modes in several $A$ and $B$ stars. The rapid rotation typical of such stars can interact with other global oscillations to produce inertial modes. Overshooting convection from the cores of hot stars can also excite a wide spectrum of $r$ - and g-modes in their radiative envelopes similar to the solar case (Wolff 1995).

\section{References}

Cohen, D.H., Cassinelli, MacFarlane, J.J. 1997a, ApJ 487, 867

Cohen, D.H., Cassinelli, Waldron, W.L. 1997b, ApJ 488, 397

Dziembowski, W.A. 1994, in Pulsation, Rotation and Mass Loss in Early-Type Stars, L.A. Balona et al. (eds), 55

Gilman, P.A., Miller, J. 1981, Ap. J. Supp. Ser. 46, 211

Israelian, G., Chentsov, E., Musaev, F. 1997, MNRAS 290, 521

Mikic, Z., Linker, J.A. 1994, ApJ 430, 898

Moss, W.A. 1994, in Pulsation, Rotation and Mass Loss in Early-Type Stars, L.A. Balona et al. (eds), 173

Morrison, N.D., Mulliss, C.L. 1998, BAAS 198

Ozaki, Y. 1986, in Seismology of the Sun and the Distant Stars, D. O. Gough (ed.), 453

Saio, H. 1982, ApJ 256, 717

Smith, M.A., Robinson, R.D., Corbet, R.H.D. 1998, ApJ 503, 877

Smith, M.A., Robinson, R. D., Hatzes, A.P. 1999, ApJ 507, 945

Tikhomolov, E.M., Mordvinov 1996, ApJ 472, 389

Tout, C.A., Pringle, J.E. 1995, MNRAS 272, 528

Van Hoven, G., Mok, Y., Mikic, Z. 1995, ApJ 440, L105

Wolff, C.L. 1995, ApJ 443, 423 